\title{
La casa di Anna e altre belle storie
}

\author{
Claudia Conforti
}

Vittorio De Feo (Napoli i928 - Roma 2002) era un architetto colto. Attratto da curiosità eccentriche e appassionato di letteratura e di arte, si innamorava di un'opera e ne parlava con gli amici. Nel r989 Barbara Briganti gli fece dono di un libretto che aveva tradotto per Sellerio: La petite maison, un racconto libertino di Jean-François de Bastide (I724-I 798) Le petites maisons nel Settecento erano i casini di delizia intorno a Parigi, dove l'aristocrazia si abbandonava al gioco d'amore e d'azzardo. La petite maison del racconto è l'arma che il marchese di Tremicour, seduttore impenitente, sfodera per conquistare Melite. La giovane aristocratica molto sicura di sé accetta la scommessa di accompagnarlo nella petite maison, convinta di resistere al fascino congiunto della casa e del marchese. Melite è una parigina con uso di mondo, ben diversa da Madelon e Cathos Gorgibus, le smorfiose provinciali, i cui sentimentalismi sono ridicolizzati da Molière nelle Précieuses ridicules (1659). Il racconto pertanto si snoda nel duplice registro di spazio e di eros. L'architettura si carica di sensualità: le sale, i cabinets, le camere, i dipinti, gli arredi, le luci, gli automi (il tavolo imbandito cala dal soffitto, per essere inghiottito dal pavimento alla fine del pasto), il giardino e le fontane, assaporati dalle parole del narratore, affascinano come sapienti carezze carnali. La schermaglia amorosa si conclude, a sorpresa, con la capitolazione di Melite, che «frémit, se troubla, soupira, et perdit la gageure».

Il racconto incantò $\mathrm{De} F$ Feo, che si riconobbe nella dimensione sensoriale e giocosa dell'architettura di Bastide. Fin dagli anni Settanta infatti egli perseguiva un'idea di architettura in controtendenza con lo schiacciante primato dell'ideologia, allora in àuge: si pensi al titolo provocatorio Il piacere dell'architettura dato da De Feo al libro in cui traeva il primo bilancio della sua opera, illustrando ventidue architetture: dal progetto vincitore del concorso (1957) per le Preture Riunite di Roma a quello per il teatro comunale di Forlì (1976), riprodotto in copertina ${ }^{2}$. In linea con il titolo, l'autore avvertiva che la scelta delle architetture «e gli spazi attribuiti dipendono soltanto dal grado di simpatia dell'autore per ciascun progetto» 3 .

De Feo si sentì sfidato a replicare il racconto libertino, ambientandolo nel suo tempo e in una sua architettura, non costruita, ma non per questo immaginaria. Anzi accuratamente progettata, tanto da illustrare l'occhietto del libro Vittorio De Feo. I3 progetti, dove nel I99I traeva un secondo bilancio del suo lavoro ${ }^{4}$. Il racconto, intitolato La casa (e Anna) fu scritto di getto, a penna stilografica, con pochissime correzioni, sulle pagine a quadretti di un minuscolo calepino, che De Feo fece rilegare in tela rossa, con impressioni in oro. L'amico Sergio Polano nel i99I ne fece una preziosa stampa fuori commercio, corredata da un'incisione sciolta a tratto bianco su carta grigia, a doppia faccia, con il progetto della casa. Sul recto uno schizzo prospettico dell'insieme esibisce il numero dell'incisione e la firma dell'autore; sul verso la pianta e un prospetto. La casa di Anna è generata da geometrie regolari (rettangoli, quadrati e un ovale che detta segretamente gli allineamenti): lievi rotazioni planimetriche provocano collisioni e incastri che sommuovono i volumi, attraversati da prospettive inattese e sfuggenti.

La pianta della casa di Anna campeggia anche sulla copertina del libro Tre racconti di ar- 

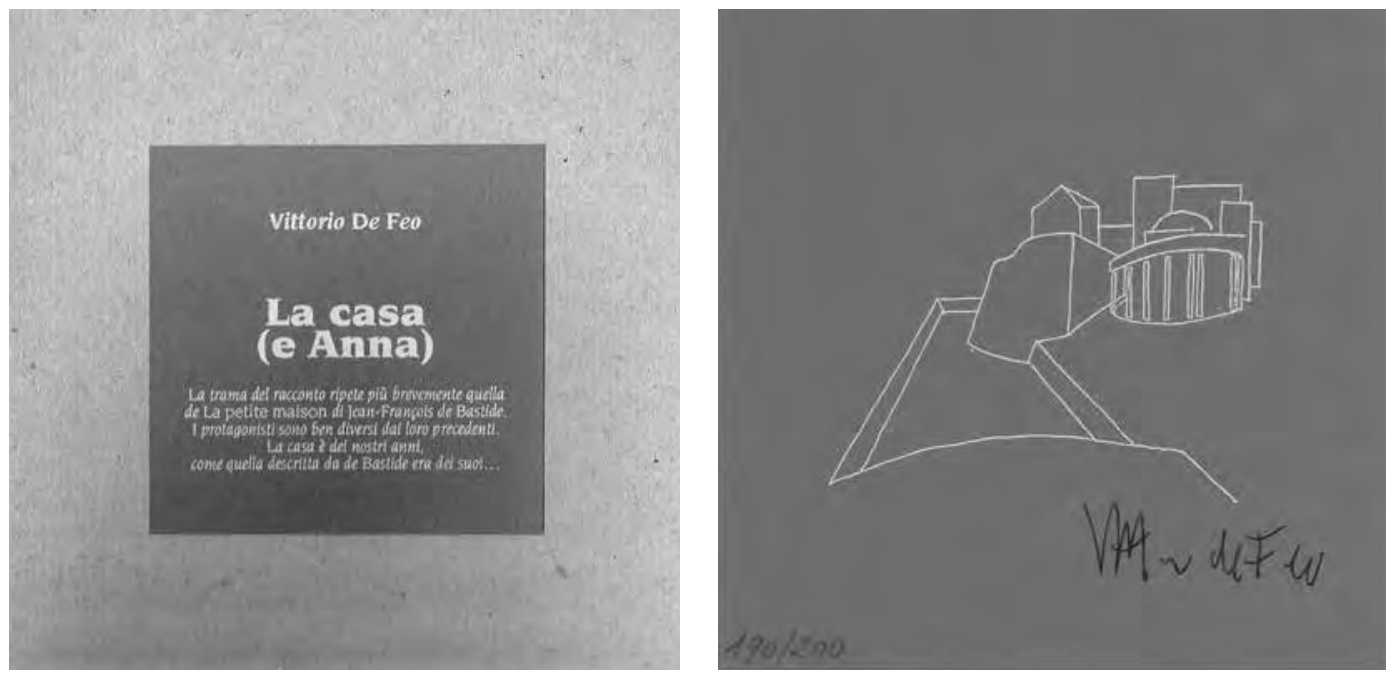

I-2. Vittorio De Feo, La Casa (e Anna), a cura di Sergio Polano, Archilibri, Venezia i99. Copertina e incisione allegata.

chitettura del 1997 , dove il racconto fu ripubblicato's. I protagonisti sono Anna, da tempo amica di Giovanni, l'architetto deuteragonista che, a fronte di una crisi esistenziale della giovane donna dall'indole anticonformista, se ne sente «irresistibilmente attratto», fino a essere «dominato da una passione che malamente riusciva a controllare» ${ }^{6}$. Ma l'abitudine amicale è d'ostacolo alla soddisfazione erotica, la cui semplice prospettiva raggela Anna. Giovanni allora fa leva sull'interesse che l'amica ha sempre dimostrato all'architettura e la invita a una visita autunnale nella sua casa di campagna. Lì, in un impalpabile crescendo, si ricrea l'intreccio tra erotismo e spazio, fino alla conclusione, venata da una strana malinconia anticipata dal clima d'autunno. Anna accondiscende infine al desiderio di Giovanni, che tuttavia «non riuscì ad esserne davvero felice, perché improvviso sentì il peso di una grande stanchezza. Gli pareva anche che avendo testimoniato in modo tanto minuzioso le proprie ragioni e visioni, potesse risultare ormai irrilevante e incolpevole porre fine alle emozioni e ai desideri» ${ }^{7}$.

Qual è dunque l'autentico oggetto del desiderio di Giovanni: la casa o Anna?

E quale aspetto del racconto di Bastide ha suscitato l'emulazione di De Feo? Dell'interpretazione dell'architettura come oggetto capace di suscitare emozioni e sensualità, abbiamo detto. Ma ho ragione di credere che sia stata determinante anche l'efficacia ecfrastica di Bastide, la cui evidenza visiva suscita iden- tificazione ed empatia con i protagonisti. $\mathrm{La}$ petite maison è un racconto che non si limita a catturare con le parole un pallido riflesso degli ambienti: la narrazione diventa un potente atto creativo, autonomo e competitivo, che dice dell'architettura più di quanto essa, da sola, non sappia dire.

È una sfida irresistibile per De Feo, dotato di un estro pervasivo che si applica con pari eleganza al disegno, al progetto, alla pittura e alla scrittura. Tra i suoi amici si contano numerosi gli artisti, spesso diversamente eccentrici. Come Toti Scialoja (I9I4-I998), a cui lo legava il comune amore per Procida, luogo d'origine del pittore, dove De Feo aveva costruito per sé un'incantevole casa di vacanza, e il gusto per i giochi di parole, le filastrocche e le rime giocose. Come Carla Accardi (1924-20I4), di cui avrebbe voluto un'opera a sfondo dell'altare maggiore della chiesa di San Tommaso d'Aquino a «Tor Vergata». Come Riccardo Tommasi Ferroni (1934-2000), di cui ammirava la straordinaria abilità grafica, la spregiudicatezza espressiva e, soprattutto, lo sradicamento temporale ${ }^{8}$. Le pitture di Tommasi Ferroni infatti, sciolte dai lacci temporali, transitano liberamente tra contemporaneo e Seicento. Un secolo questo che De Feo predilige, di cui perlustra i meandri più tortuosi $\mathrm{e}$ sfuggenti, che fanno fiorire la materia narrativa del racconto In memoria e ordiscono i suoi saggi magistrali sul barocco: La Piazza del Quirinale (1973) e le appassionate, reiterate, esegesi di Andrea Pozzo (1642-1709)9. Anche 


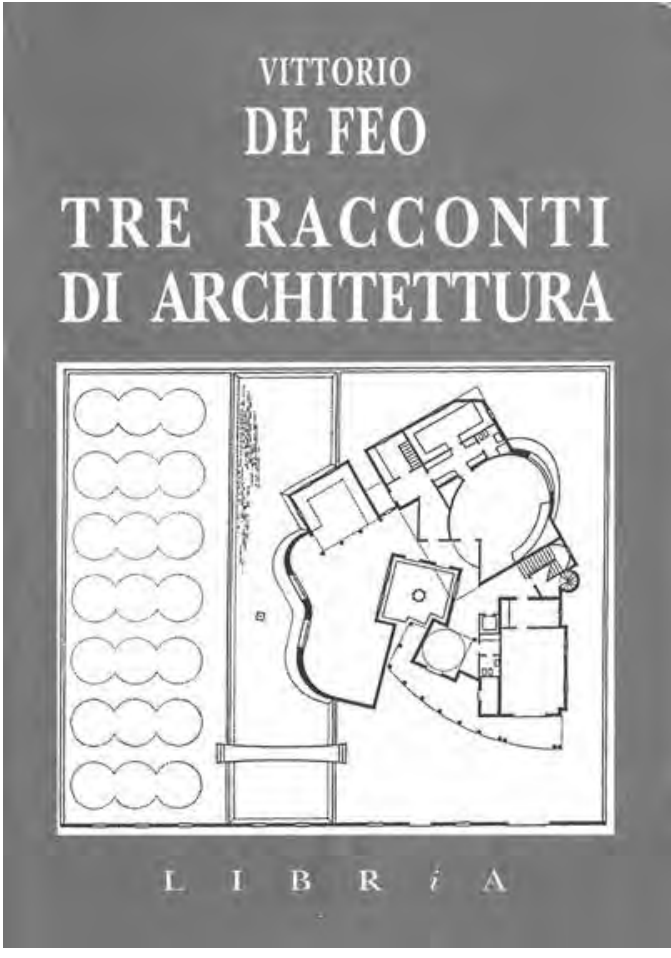

3. Vittorio De Feo, Tre racconti di architettura, Edizioni Libria, Melfi 1997. Copertina.

le prove pittoriche di De Feo meritano perspicua attenzione per la perizia tecnica, la sperimentazione figurativa e l'esattezza espressiva al servizio dell'architettura. La più celebre delle sue tavole, con la prospettiva rombante della stazione di servizio Esso (I970), è assurta a icona dell'intreccio che nella Roma degli anni Sessanta-Settanta stringeva l'architettura alle arti sorelle ${ }^{\mathrm{I}}$.

Catturato dalla rete che impiglia le arti visive alla parola, De Feo scrive per gli amici pungenti nonsense, gentili apologhi, minuscoli memoriali e amabili racconti, tre dei quali sono quelli pubblicati nel 1997: La casa (e Anna), La committenza, In memoria. Arabeschi letterari con laconici inserti grafici (due nei primi e uno nell'ultimo) che celano più di quanto rivelino.

La committenza allude a un potenziale incarico di progetto: il protagonista è ancora Giovanni, l'architetto di Anna, del quale apprendiamo che il cognome è Antinori. Anche questo racconto è corredato da due disegni dell'autore: una sala da pranzo della Hofburg, stilizzata in un interno borghese napoletano, e una vignetta di Tintin, l'e-

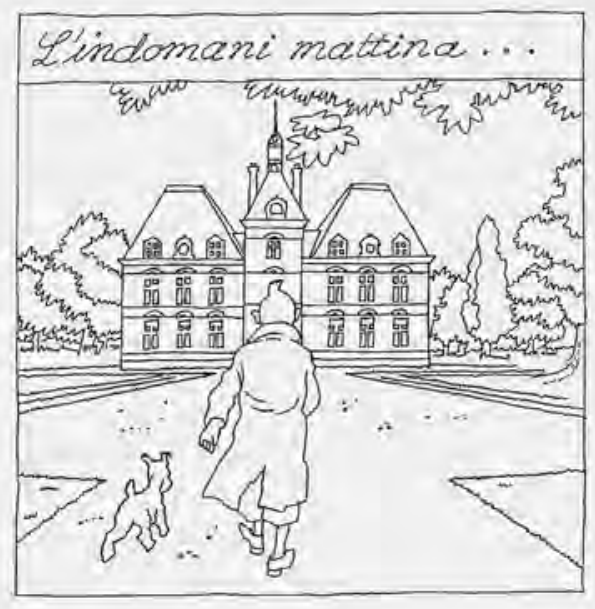

Tin Tine le sette ifore di cristallo.

4. Tintin e Milou, in Vittorio De Feo, Tre racconti di architettura, Edizioni Libria, Melfi I997, p. 40.

roe dei fumetti di Hergé, colto di spalle con il fido Milou mentre marcia gagliardamente verso uno château che domina la composizione $^{11}$. In questo testo l'ecfrasi si esercita su i piatti opulenti della cucina napoletana, in un crescendo di profumi, consistenza e colori che fa dimenticare l'oggetto del racconto, l'ipotetica committenza di una villa di campagna, e accende l'immaginazione gastrica del lettore. Il terzo racconto, In memoria, è enigmatico ed eccentrico. Qui non si tratta di un esercizio ecfrastico, ma di una ricerca storica di esemplare rigore metodologico, rivolta a un bizzarro edificio di via dei Cerchi a Roma: una quinta a intonaco di pozzolana grigio violaceo, traforata da un portale e da finestre di varia geometria, tutte profilate sinuosamente a stucco piatto e fiorite da gigli farnesiani. La spaesata quinta architettonica aveva colpito, anni prima, anche l'immaginazione del pittore milanese Gianfilippo Usellini (1903-197I) che nel I947 la dipinse nel quadro La casa degli amorini. Tramite la denominazione l'architettura viene apparentata all'omonima domus pompeiana dall'artista, che vi dissemina giocondi putti affacciati alle 


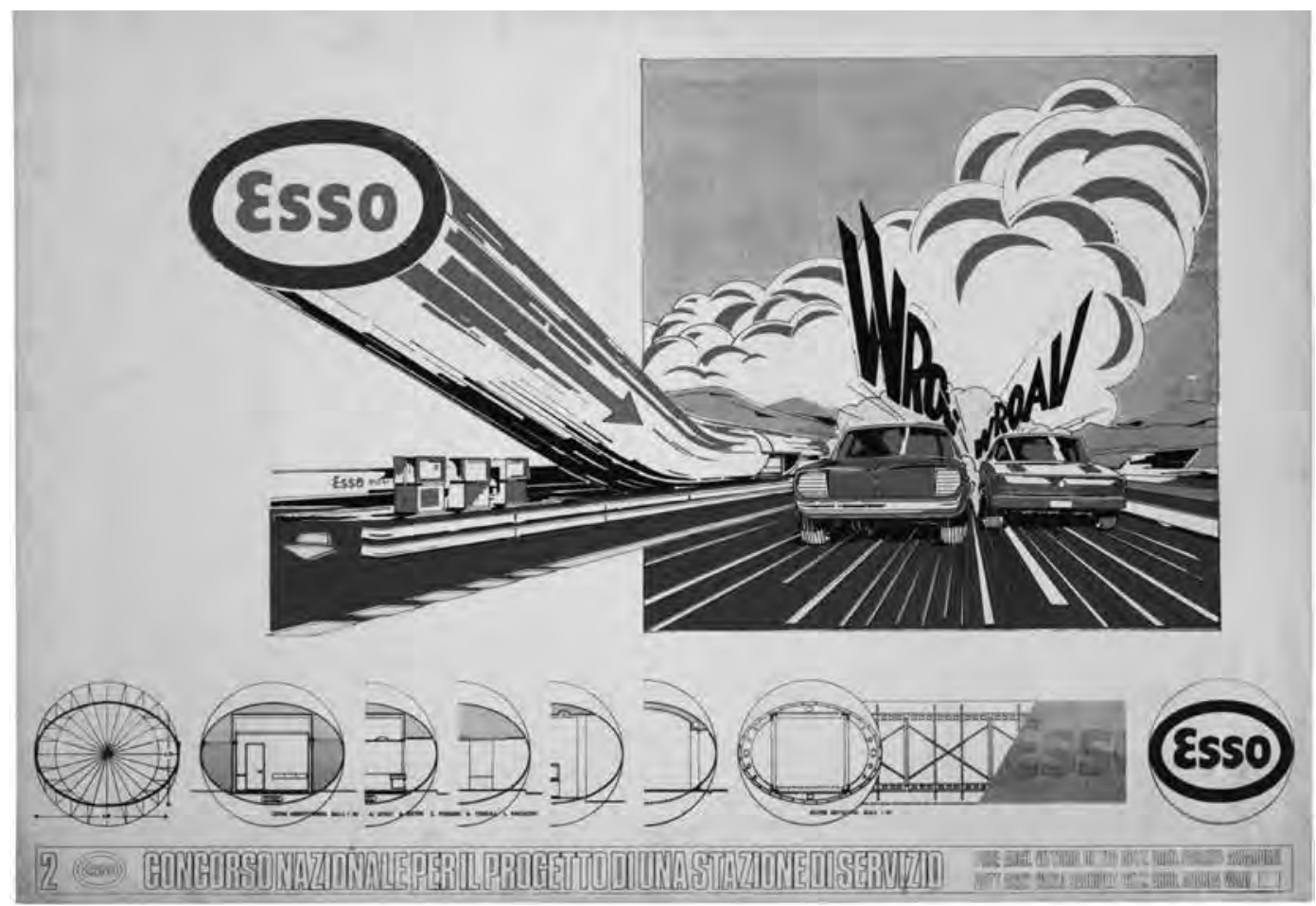

5. Vittorio De Feo, con Fabrizio Aggarbati, Carla Saggioro, Andrea Vigni, concorso per una stazione di servizio tipo della Esso, I970, primo premio. Tavola prospettica.

finestre e agli oculi della cimasa. Il quadro era probabilmente conosciuto da De Feo, che amava quel genere di pittura giocosa e surreale, ed era assiduo della Galleria dell'Oca che, in via dell'Oca, alle spalle dello studio di via Brunetti, nel I98 I, a dieci anni dalla morte del pittore, allestì la mostra: Gianfilippo Usellini. Tempere grasse all'uovo secondo la ricetta degli antichi maestri, in cui il quadro era esposto $^{\text {I2 }}$. L'opera di Usellini colloca l'architettura ai confini con i territori della creatività, di cui essa rivela percorsi sconosciuti, dove i sogni e la memoria agitano finalità metafisiche. Non diversamente da quanto rivelano le figurazioni immote e silenti con cui De Feo accompagna i suoi progetti. Usellini, con la sua tempera, evidenzia il carattere atemporale e surreale, lo stesso che colpisce De Feo, di quell'architettura «coronata da un lento arco rovescio traforato a margine di una sequenza di oculi ${ }^{13}$. Il lento arco rammentato da De Feo è una catenaria, figura geometrica la cui scoperta si fa risalire a Galileo Galilei ${ }^{\mathrm{I}}$. Attenzione: l'annotazione non è uno sfoggio di erudizione, come vedremo! Al centro della catenaria si protende al cielo una mano de- stra: calco in gesso di un originale perduto, forse antico forse moderno, in origine $\mathrm{cu}$ stodito in una chiesetta denominata da quel reperto Santa Maria de Manu e demolita da Sisto V intorno al is 87 per tracciare la strada lungo il Circo Massimo. Con l'indice puntato al cielo, la mano sembra additare il mondo delle sfere celesti, la cui investigazione costò cara a Galileo. La medesima mano in stucco contrassegnava anche il vertice delle cornici delle due finestre laterali dell'ultimo piano, dove sopravvive in quella di destra. De Feo, accertata l'assenza di documenti diretti sulla costruzione (dovuta a suo avviso alla sistematica e volontaria distruzione, perseguita per somma prudenza dal committente), collaziona innumerevoli e disparati indizi, grazie ai quali approda all'identificazione del committente. Costui sarebbe «Giovanni Castelli medico chirurgo e professore alla romana Sapienza» ${ }^{15}$. Di Giovanni (ancora quel nome!) De Feo ricostruisce diramate ascendenze, la frequentazione di circoli scientifici e spiritualisti, chiamati segretamente "giordanisti»: si sussurrava infatti, tra Roma e Napoli, che essi onorassero clandestinamente la memoria 


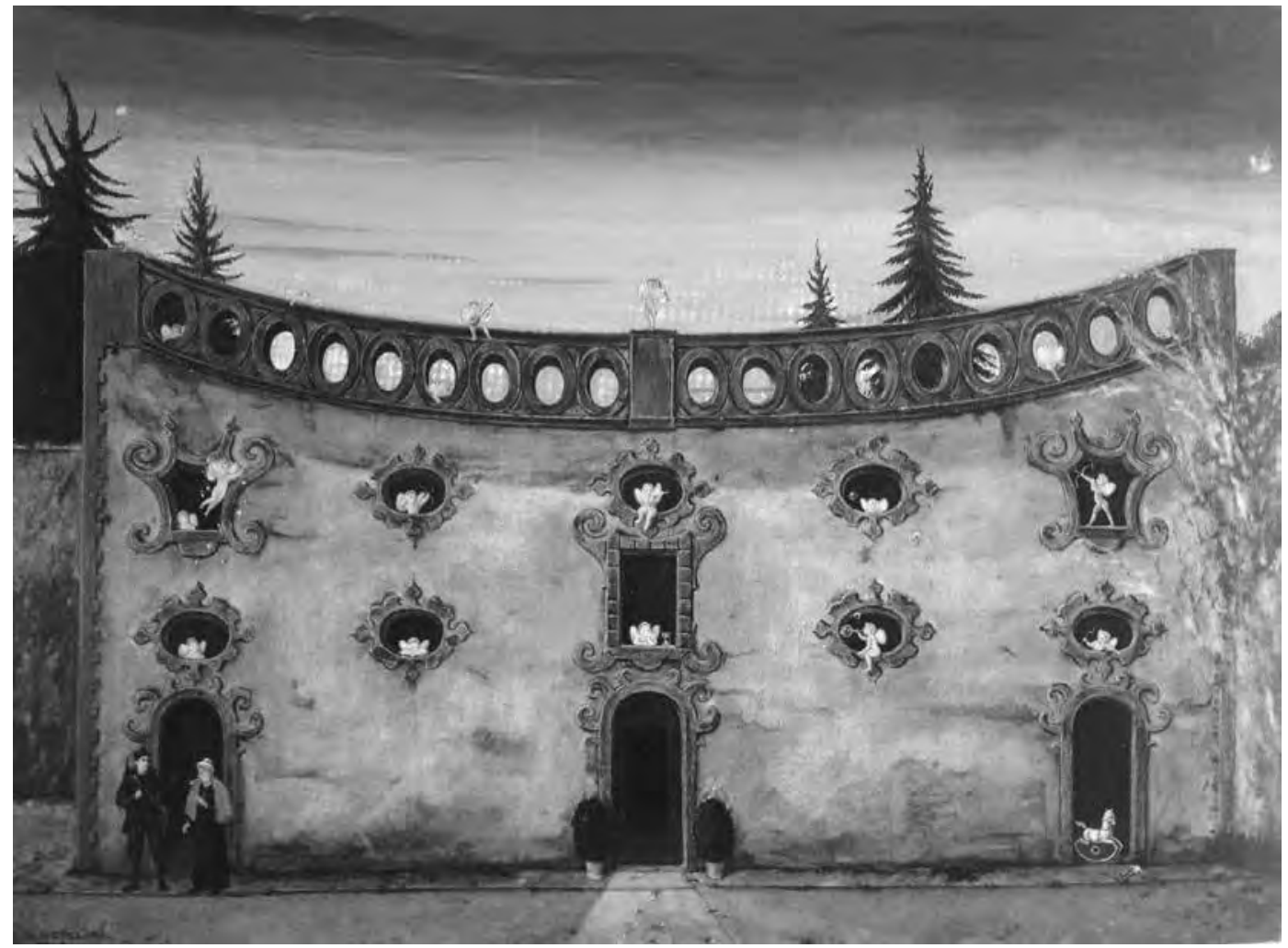

6. Gianfilippo Usellini, La casa degli amorini, I947.

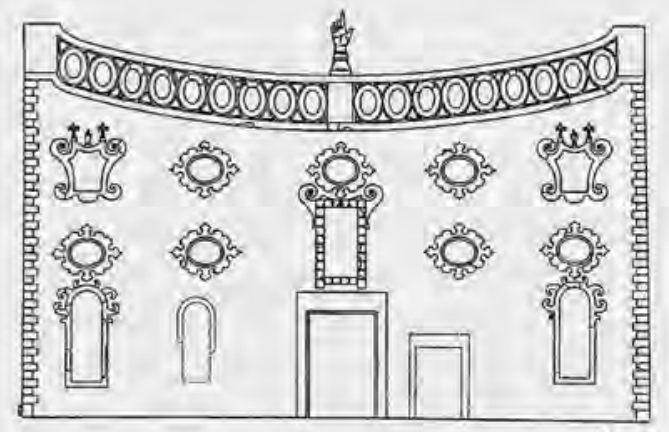

Casa m via dei Cenchĩ a Roma

7. La casa di via dei Cerchi in Vittorio De Feo, Tre racconti di architettura, Edizioni Libria, Melfi I997, p. 44. di Giordano Bruno, giustiziato e bruciato per eresia da Clemente VIII nel i600. Il Castelli era affittuario di casupole alle falde del $\mathrm{Pa}$ latino, nei giardini del cardinale Farnese, del cui celebre orto botanico fu direttore, ricorda De Feo, proprio il celebre bisavolo di Giovanni, Pietro Castelli (I 575?-I66I). Pietro Castelli è storicamente vissuto: fu professore di botanica alla Sapienza, ma non diresse il viridario farnesiano sul Palatino, bensì, sotto mentite spoglie, ne redasse un compendio, illustrato da sue incisioni della flora esotica del giardino. Fondatore dell'Orto Botanico di Messina, Pietro morì in tarda età e fu sepolto nella città siciliana ${ }^{16}$. Il racconto di De Feo è trapunto da divagazioni di questo tipo, in cui la verità storica stinge in invenzioni plausibili, configurando un labirinto dove si muovono personaggi storicamente attestati, come Gottfried Wilhelm Leibniz, colto nel soggiorno romano del I689; il medico professore dell'Alma Mater di Bologna Marcello Malpighi; i filosofi scienziati Girolamo Brasavoli e Lucantonio Porzio, professore allo studio napoletano e amico di Giovan Battista 


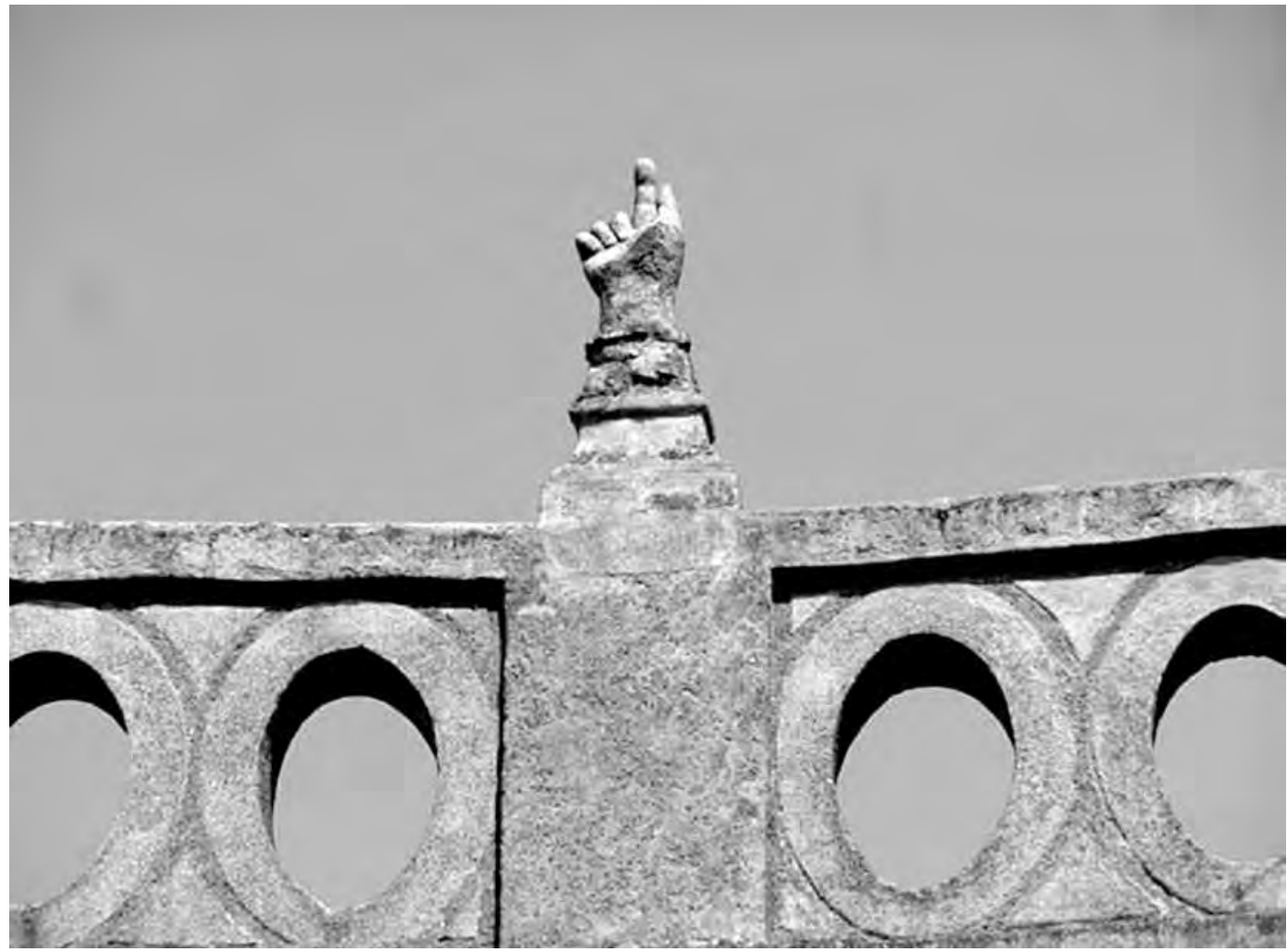

8. La mano che punta al cielo nel coronamento della casa di via dei Cerchi a Roma, fine XVII secolo.

Vico; Giovanni Giustino Ciampini, direttore del "Giornale de' Letterati», su cui pubblicò anche Porzio. Mescolati ai personaggi storici si aggirano personaggi d'invenzione, come Giovanni e la matura nobildonna Gerica Porzio, amica di penna del protagonista, le cui lettere forniranno al ricercatore De Feo la chiave dell'enigma. Altri personaggi ancora sono esistiti, ma erano già morti all'epoca dei fatti narrati, come il cardinale Francesco Maria Farnese (I6I9-1647). In una supplica a lui indirizzata, citata da De Feo con tanto di fonte (apocrifa), il Castelli chiede il permesso di «alzare facciata, che adesso amancha» davanti alle casupole, così da comporre dignitosamente il fronte su strada della proprietà ${ }^{17}$. La richiesta sembra innocente. Non lo è. Essa mira a un obiettivo tanto pericoloso quanto inconfessabile, che giustifica il titolo In memoria. Criptiche allusioni che Gerica affida ad alcune lettere a Giovanni indirizzano lo studioso verso lo scioglimento dell'enigma. La misteriosa facciata che punta l'indice della mano destra al cielo è un monumento che, a distanza di un secolo, nel I700, rende segre- tamente omaggio al sacrificio di Giordano Bruno e alla sua filosofia, che attinge a «una verità così grande, che non si può rappresentarla se non per immaginazione, figurata con simboli nuovi» ${ }^{18}$.

\section{Note}

Il racconto, ispirato al Pavillon de La Boissière, ovvero all'Hôtel de La Bouëxière, costruito per l'esattore fiscale Jean Gaillard de La Bouëxière intorno alla metà del XVIII secolo da Antoine Matthieu Le Carpentier (I709-I773), fu pubblicato dapprima (I760) su un periodico, poi nel 1763 nella raccolta Contes Moraux, 4 voll., L. Cellot, Paris 1763 , vol. I, pp. 47-88; sull'autore si veda K. Astbury, Les contes de Jean-François Bastide, in M. Cook, M.E. Plagnol-Diéval (éds.), Anecdotes, fait-divers, contes, nowvelles $1700-1820$, Peter Lang, Bern 2000, pp. I 47-I 58.

2 Il volume uscì nella pionieristica collana di architettura di Francesco Moschini, «Città e progetto», Editrice Magma, Roma 1976, con Presentazione dello stesso Moschini e Introduzione di Francesco Dal Co.

3 V. De Feo, Il piacere dell'architettura, Editrice Magma, Roma I976, p. 23. Sull'architettura di De 
Feo si vedano C. Conforti, F. Dal Co, Vittorio De Feo. Opere e progetti, Electa, Milano i986; V. De Feo, I3 progetti, con presentazione di M. Zardini, Officina Edizioni, Roma I99I.

4 Vittorio De Feo cit., occhietto, verso, la didascalia recita: «Casa per A.N.», le iniziali stanno per Anonimo Napoletano, una sigla che De Feo usava per firmare le sue creazioni extraprofessionali. Mi sono chiesta se questo divertissement letterario e architettonico abbia anche il senso di un omaggio segreto all'ammiratissimo Le Corbusier, che nel I954 pubblicò a Zurigo con l'editore Girsberger il libretto Une petite maison, 1923 , dedicato alla casa di famiglia sul lago Lemano.

s V. De Feo, Tre racconti di architettura, Libria, Melfi i 997 .

6 V. De Feo, La casa (e Anna) in ivi, p. Io.

7 Ivi, p. 23.

8 Tra settembre 1998 e gennaio I999, in occasione di una mostra al Museo di Arte Sacra di Camaiore, intitolata Leonardo e la Pulzella di Camaiore. Inediti vinciani e capolavori della scultura lucchese del primo Rinascimento, il curatore, insigne studioso del Da Vinci, Mario Pedretti, riconobbe come opera di Leonardo il disegno di un Cavallo impennato con cavaliere nudo, realizzato in gioventù da Riccardo Tommasi Ferroni.

9 Vittorio De Feo, La piazza del Quirinale, Officina Edizioni, Roma 1973; il primo scritto su Pozzo è datato I980: L'architettura immaginata da Andrea Pozzo gesuita, «Rassegna di Architettura e Urbanistica», 46, I980; l'ultimo Andrea Pozzo in A. Scotti (a cura di), L'architettura Italiana, Il Seicento, Electa, Milano 2003, pp. 262-277.

1o La tavola, esposta in più occasioni al MAXXI, è stata ripetutamente pubblicata a corredo degli scritti su De Feo. Sugli intrecci tra l'architettura e le arti in Italia nel XX secolo si veda il catalogo della mostra Arte e Architettura. Punti di vista, Mantova 5 maggio - 5 giugno 2016, Corraini, Mantova 2016, in particolare R. Dulio, Architetti Pittori, pp. 7076, dove è pubblicato il quadro di De Feo Ab Ovo. Ritratto di un'amica (1985).

II Il ragazzino Tintin, affiancato dal bianco Fox terrier Milou, è protagonista delle bandes dessinées del belga George Remi (1907-1983), in arte Hergé; le storie di Tintin hanno accompagnato la fanciullezza di De Feo, che ne apprezzava il segno sintetico e gli squillanti colori timbrici. Un album di Tintin risalente all'infanzia dell'architetto è conservato nella biblioteca di De Feo al MAXXI.

12 La Galleria dell'Oca, diretta da Luisa Laureati Briganti, inaugurò la mostra il 26 febbraio I98I (www. luisalaureatibriganti.it/mostre/archivio8o.html). Il quadro, una tempera su tela $98 \times 130 \mathrm{~cm}$, firmato e datato 1947, è documentato nella scheda n. 37 di F. Usellini e L. Sansone, in Elena Pontiggia (a cura di), Gianfilippo Usellini I903-I97I, catalogo della mostra (Sondrio, 1994), Leonardo Arte, Segrate 1994, p. I84; una riproduzione dell'opera è pubblicata in ivi, a p. 82. Sono grata a Roberto Dulio per la segnalazione.

13 V. De Feo, In memoria, in Id., Tre racconti di architettura cit., p. 45 .

${ }^{14}$ G. Galilei, Discorsi e Dimostrazioni Matematiche Intorno a Due Nuove Scienze attenenti alla mecanica e i movimenti locali, Apresso gli Elzevirii (Elezevier), Leida I638. Nella seconda e nella quarta giornata, Filippo Salviati (I 57 I-I 620), astronomo e nobile fiorentino, tra gli interlocutori del dialogo sulla fisica terrestre, illustra l'esistenza e la forma della catenaria.

is V. De Feo, In Memoria cit., p. 46.

${ }_{16}$ Tobia Aldinus, Exactissima descriptio rariorum quorundarum plantarum quae continentur Romae in horto Farnesiano, Typis Jacobi Mascardi, Roma I625.

17 V. De Feo, In Memoria cit., p. 48: il cardinale in questione è esistito, ma è morto assai prima degli avvenimenti narrati da De Feo.

${ }^{18}$ V. De Feo, Tre racconti di architettura cit., p. 55. 